\title{
A simple cognitive task intervention to prevent intrusive memories after trauma in patients in the Emergency Department: A randomized controlled trial terminated due to COVID-19
}

Marie Kanstrup ${ }^{1,2^{*}+}$, Laura Singh L, $^{3 *+} \mathbb{D}$, Katarina E. Göransson ${ }^{5,6}$, Beau Gamble ${ }^{3}$, Rod S. Taylor ${ }^{7}$, Lalitha lyadurai ${ }^{8}$, Michelle L. Moulds ${ }^{9}$ and Emily A. Holmes ${ }^{1,3}$

\begin{abstract}
Objective: This randomised controlled trial (RCT) aimed to investigate the effects of a simple cognitive task intervention on intrusive memories ("flashbacks") and associated symptoms following a traumatic event. Patients presenting to a Swedish emergency department (ED) soon after a traumatic event were randomly allocated (1:1) to the simple cognitive task intervention (memory cue + mental rotation instructions + computer game "Tetris" for at least $20 \mathrm{~min}$ ) or control (podcast, similar time). We planned follow-ups at one-week, 1-month, and where possible, 3- and 6-months post-trauma. Anticipated enrolment was $N=148$.

Results: The RCT was terminated prematurely after recruiting $N=16$ participants. The COVID-19 pandemic prevented recruitment/testing in the ED because: (i) the study required face-to-face contact between participants, psychology researchers, ED staff, and patients, incurring risk of virus transmission; (ii) the host ED site received COVID19 patients; and (iii) reduced flow of patients otherwise presenting to the ED in non-pandemic conditions (e.g. after trauma). We report on delivery of study procedures, recruitment, treatment adherence, outcome completion (primary outcome: number of intrusive memories during week 5), attrition, and limitations. The information presented and limitations may enable our group and others to learn from this terminated study.
\end{abstract}

Trial registration ClinicalTrials.gov: NCT04185155 (04-12-2019)

Keywords: Terminated study, Emergency Department, COVID-19, Intrusive memories, Psychological trauma, Prevention, RCT, Behavioural intervention

*Correspondence: marie.kanstrup@ki.se; laura.singh@psyk.uu.se ${ }^{\dagger}$ Marie Kanstrup and Laura Singh co-shared first authorship

${ }^{1}$ Division of Psychology, Department of Clinical Neuroscience, Karolinska Institutet, 17177 Stockholm, Sweden

${ }^{3}$ Department of Psychology, Uppsala University, Box 1225, 751

42 Uppsala, Sweden

Full list of author information is available at the end of the article

\section{Introduction}

This RCT (ClinicalTrials.gov: NCT04185155) aimed to investigate the effects of a simple cognitive task intervention on intrusive memories ("flashbacks") and other symptoms in patients presenting to a hospital Emergency Department (ED) in Sweden (part of the public healthcare system for all individuals in need of medical emergency care) soon after a traumatic event. Anticipated

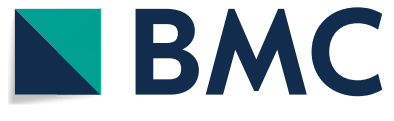

c The Author(s) 2021. This article is licensed under a Creative Commons Attribution 4.0 International License, which permits use, sharing, adaptation, distribution and reproduction in any medium or format, as long as you give appropriate credit to the original author(s) and the source, provide a link to the Creative Commons licence, and indicate if changes were made. The images or other third party material in this article are included in the article's Creative Commons licence, unless indicated otherwise in a credit line to the material. If material is not included in the article's Creative Commons licence and your intended use is not permitted by statutory regulation or exceeds the permitted use, you will need to obtain permission directly from the copyright holder. To view a copy of this licence, visit http://creativeco mmons.org/licenses/by/4.0/. The Creative Commons Public Domain Dedication waiver (http://creativecommons.org/publicdomain/ zero/1.0/) applies to the data made available in this article, unless otherwise stated in a credit line to the data. 
enrolment was $N=148$. Primary outcome was the number of intrusive memories of the traumatic event (week 5), recorded using a 7-day diary. We predicted that compared to attention placebo, participants receiving the intervention would develop fewer intrusive memories, and less severe related clinical symptoms. We also planned to explore implementation and training aspects in a hospital context. The study was preceded by a pilot RCT (ClinicalTrials.gov: NCT03509792) in the same ED $(N=41)[1]$.

The simple cognitive task intervention targets intrusive memories of trauma (recurrent, distressing memories that spring to mind unbidden). They are a core clinical feature $[2,3]$ of posttraumatic stress disorder (PTSD) [4]. Targeting intrusive memories is important in its own right to alleviate distress $[3,5]$, hence the choice of the number of intrusive memories as the primary outcome [6]. Reducing early intrusions might also reduce the risk of developing PTSD [7], and help reduce related clinical symptoms. The intervention comprises a single session with several components: a brief memory reminder cue (to activate the trauma memory), followed by a visuospatial cognitive interference task (playing the computer game 'Tetris' on smartphone for at least 20 min following training to use 'mental rotation' throughout gameplay [1]). The rationale behind the intervention $[8,9]$ and more details about the procedure are described elsewhere $[1,5]$. Patients are not required to talk about the trauma in detail. The single session (c. $30 \mathrm{~min}$ ) takes place with a researcher in the ED whilst patients are waiting for medical care. They can engage in self-administered 'booster' sessions remotely thereafter. The attention placebo control condition was listening to a podcast (on smartphone) $[1,10]$ for a similar duration.

Participants were recruited in the ED [11] within c. $6 \mathrm{~h}$ of experiencing a traumatic event [6]. As often the first port of call after a traumatic event, the ED provides a setting in which a preventive intervention can be evaluated. It enables recruitment of participants with mixed trauma types (e.g., motor vehicle accidents, industrial accidents), and provides an opportunity to evaluate an intervention to trauma-exposed individuals in the first few hours post-trauma. Our pilot work indicated that ED patients reported intrusive memories following traumatic events that ranged from minor fall accidents to severe injuries [1].

The current RCT was halted prematurely (10-07-2020) due to the COVID-19 pandemic, after only $N=16$ participants were randomised. The pandemic prevented recruitment/testing in the ED as it placed significant demands on ED resources and introduced risk of potential infection (due to face-to-face meetings between participants, psychology researchers, ED staff, and patients during study procedures). The host ED was used to receive COVID-19 patients and had a reduced flow of the type of patients who would otherwise present to the ED after trauma in non-pandemic conditions. Many clinical trials internationally have been terminated due to the COVID-19 pandemic [12, 13]. Despite termination, we report information about delivery of study procedures (methods), recruitment rates, treatment adherence, outcome completion, attrition, and limitations focusing on pandemic conditions. Our objective is to enable researchers to learn from this experience.

\section{Main text \\ Methods \\ Planned sample size}

The planned sample size $(N=148)$ was informed by a pilot RCT $(N=41)$ [1]. Based on the observed betweengroup difference of $d=0.57$ for the number of intrusive memories at week 5 (7-day diary), at power of $90 \%$ and alpha of 0.05 , we would require a sample size of $N=65$ per group (130 in total). With attrition calculated at $14.2 \%$, we aimed to recruit $N=148$ in total. ${ }^{1}$

\section{Participants}

Eligibility criteria included: having experienced or witnessed a traumatic event resulting in admission to the $\mathrm{ED}$, and able to be seen in the ED within approximately $6 \mathrm{~h}$ after the traumatic event (day 1); see ClinicalTrials. gov: NCT04185155 for full list.

\section{Procedure}

Study procedures were carried out by research assistants (RAs) supervised by clinical psychologists (MK/EAH). Session-1 was conducted entirely in the ED (e.g., waiting room/corridors-not separate rooms) on the day patients presented to the ED (day 1). Study procedures were fitted into patients' time spent in the ED (i.e. whilst waiting for medical care). Potentially eligible patients were identified in collaboration with ED staff, and in consultation with supervisors; patients received further information, and provided written and informed consent. After completing baseline assessments, participants were randomised (parallel assignment, 1:1 ratio) to intervention/control arm using a randomization tool (in electronic data collection platform accessed by RAs away from participants) which used permuted block randomization with random block sizes of 2-10. Participants were not told the condition

\footnotetext{
1 There is a discrepancy of $N=2$ between the $N=148$ in the CTR (NCT04185155) and the $N=146$ reported in (1), due to calculations being based on either $N=42$ participants randomized or $N=41$ final sample for analysis. $\mathrm{N}=146$ is based on the corrected attrition rate of $12.2 \%$ detected during the review process for (1).
} 
(intervention/control) to which they were randomized. Researchers in the ED were not blind to condition as they delivered study procedures; however, the outcome assessor was blind. Participants completed their assigned task and received information about how to complete primary and secondary outcomes. Participants in the intervention arm were contacted on day 2 and offered support for self-administered 'booster' doses of the intervention as necessary, to target intrusive memories occurring on day 2 onwards. The study was monitored by an independent clinical trial monitoring unit (Karolinska Trial Alliance). We aimed for the study to adhere to CONSORT guidelines.

\section{Planned outcomes}

Primary outcome was the number of intrusive memories of the traumatic event recorded by participants in a daily diary (morning, afternoon, evening, night) for 7 days during week 5 (5th week after Session-1). This symptom count diary has been used in our previous work $[1,6,15]$. Secondary outcomes included the number of intrusive memories in the daily diary during week 1 (i.e. in the week immediately following Session-1), and symptoms of post-traumatic stress, anxiety and depression (see ClinicalTrials.gov: NCT04185155 for details).

\section{Training to deliver intervention/control}

Two RAs who delivered the intervention/control task had received training in the pilot study [1]. A third RA only observed and provided administrative support. All RAs received training refreshers before the current trial (e.g., via role-play). Training involved several stages including the RA observing an expert (someone experienced in intervention/control delivery); RA delivering Session-1 procedures to an expert with feedback; real-time/in vivo observation by supervisors of RA with a participant until satisfactory standard; and independent intervention/control delivery by the RA with another RA present. Since RAs had psychology backgrounds (not trained hospital staff), training included guidance on how to work in the ED; e.g., hand hygiene, use of hospital uniform, how to work on a ward without getting in the way [16].

\section{Results}

\section{Participant recruitment}

This RCT was registered on ClinicalTrials.gov on 04-122019. Recruitment occurred between 10-12-2019 and 09-03-2020 in the ED (Stockholm, Sweden). Twentyeight days were spent recruiting in the ED. A supervisor was on site part of the day for 13 of these days for realtime/in vivo supervision, and available for remote supervision otherwise. Of 51 patients approached in the ED and assessed for eligibility, 27 (53\%) were excluded for not meeting eligibility criteria assessed prior to informed consent (see Fig. 1: CONSORT participant flow diagram). Of 24 potentially eligible patients offered study participation, 4 (17\%) declined to take part, 4 (17\%) were excluded prior to randomisation, and $16(67 \%)$ were randomized into the study (Fig. 1). Table 1 shows brief demographics and trauma types.

\section{Treatment adherence}

All participants completed their assigned tasks (intervention/control) in the ED in Session-1 (no drop-outs), indicating high acceptability of both arms. Three participants in the intervention condition received remote 'booster' sessions via phone to target remaining intrusive memories, which proved possible practically and acceptable to participants.

\section{Outcome completion and attrition}

Completion rates of the intrusive memory diary were $75 \%$ for week 5 (primary outcome), and $94 \%$ for week 1 (secondary outcome). Completion of other secondary outcomes (electronic platform) were $75 \%$ at one-week, $69 \%$ at 1 -month, $63 \%$ at 3 -months and $38 \%$ at 6 -months (note: the electronic platform was disabled prior to six participants' 6-months follow-up; of those who received 6 -months follow-ups, $60 \%$ completed). The main reason for attrition was that participants could not be contacted. No adverse events related to study procedures were reported. No serious adverse events were reported.

\section{Trial termination: COVID-19 pandemic prevented recruitment/testing in ED}

As the impact of the pandemic became apparent, we ceased recruitment/testing in the ED. We followed developments in the pandemic and as the situation worsened in Sweden, it was clear that continuation of the RCT would not be feasible. We decided to prematurely terminate the study on 10-07-2020. The electronic platform was disabled by the clinical trial monitoring unit on 29-07-2020. The sample $(N=16)$ comprises $11 \%$ of anticipated enrolment $(N=148)$. Thus, the terminated study data are insufficient to draw meaningful inferences for between-group comparisons e.g. regarding intervention efficacy. The full RCT was designed to provide $90 \%$ power to detect a medium effect size $(d=0.57$ observed in pilot RCT), and the current sample of $N=16$ would provide only around $19 \%$ power to detect similar effect sizes. Conducting underpowered analyses is not recommended as it (i) lowers the chance of a positive result being true (lowers positive predictive value), (ii) lowers the chance of discovering a true effect, and (iii) inflates the estimate of effect size when a true effect is discovered 


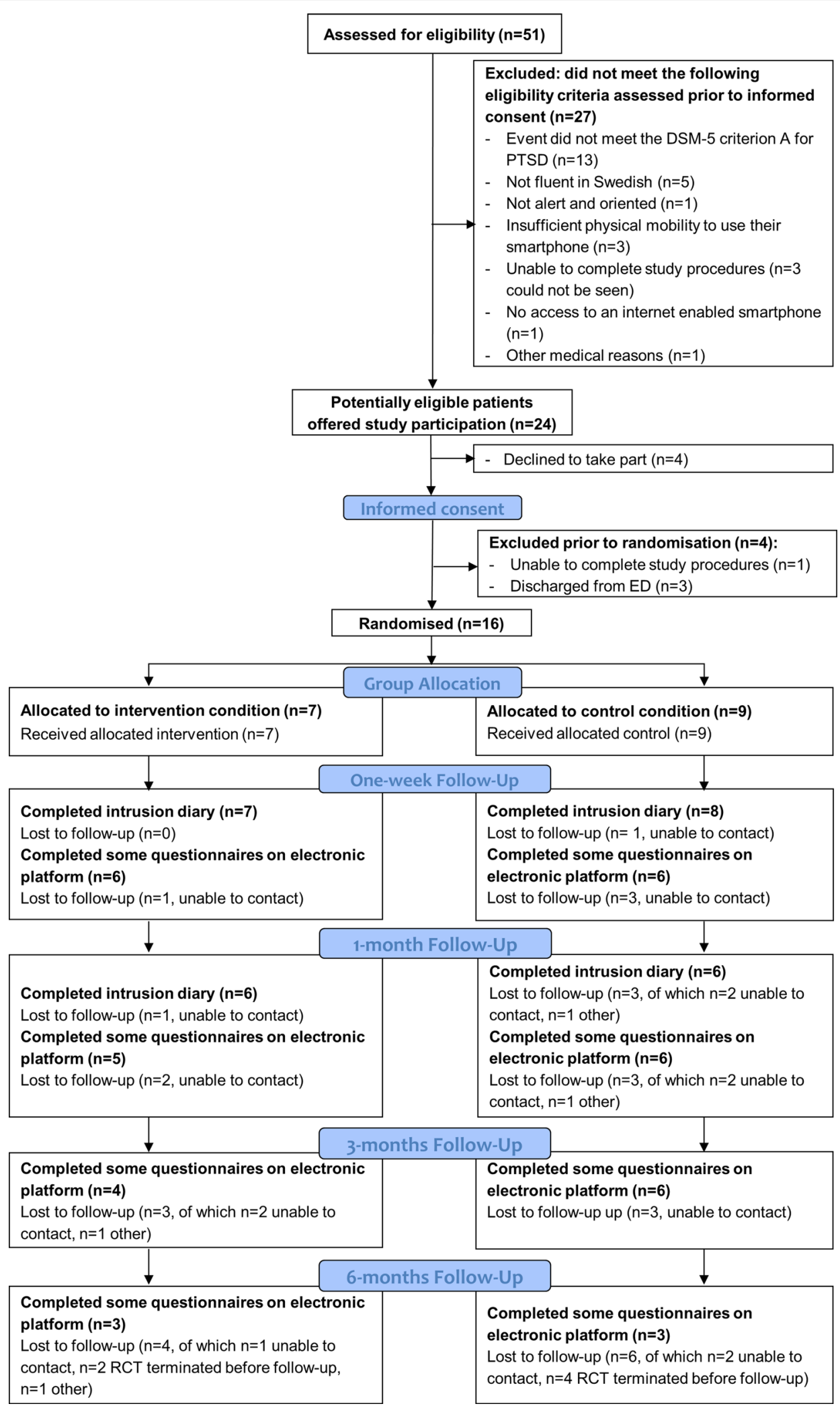

Fig. 1 CONSORT participant flow diagram 
Table 1 Brief demographics and trauma types of full sample.

Full sample $(N=16)$

Gender

Female

Male

$n=9$

Other options: Transfemale, Transmale, Genderqueer/non-binary, other identity (option to describe)

$n=7$

Age

$n=0$

$M=40.63$

$S D=14.45$

Type of trauma ${ }^{a}$

Transportation accident (e.g., car accident, bicycle accident)

$n=6$

Serious accident at work, home, or during recreational activity (e.g., slip-and-fall injury, threat to limb/extremity)

$n=10$

a The traumatic event leading to ED admission classified using Life Event Checklist 5, LEC-5

[17]. We thus conducted no formal analyses on planned group comparisons and data are not reported.

\section{Discussion}

The terminated study indicates feasible recruitment, albeit based on a small sample. Only $17 \%$ of patients approached who were potentially eligible and were offered study participation declined. Some other studies in the ED (after trauma) report a higher proportion of eligible patients declining to participate (e.g. 88\%-psychotherapy trial, 58\%-pharmacological trial [18]). All seven participants randomized to the intervention completed treatment, indicating high acceptability (treatment discontinuation is used to indicate acceptability in [19]); likewise all completed the control condition.

The team had spent months learning about and adapting to working in the ED, and adopting ED standard hygiene/safety procedures under 'normal' circumstances, i.e. establishing a sense of embeddedness of the research team in the ED environment and giving researchers the opportunity to understand the ED context, as well as potential obstacles to and facilitators of recruitment and intervention delivery. Integration of psychology research staff into the ED environment in this trial and in our pilot [1] highlight how psychological researchers can successfully work in the ED alongside ED staff in non-pandemic conditions. However, the pandemic brought another level of challenges of being in the ED to which the team could not adapt.

Exposure to traumatic events during the COVID19 pandemic appeared a rising concern for healthcare staff. Thus, we noted the need for an intervention for healthcare staff exposed to traumatic events as part of their work. Working closely with staff within the hospital had allowed us to establish collaborations and gain insights. Wellbeing of healthcare staff became a priority as the pandemic unfolded [20]. Accordingly, following termination of the current trial, we swiftly adapted study procedures for remote delivery and commenced a new trial (ClinTrials.gov: NCT04460014) targeting intrusive trauma memories in healthcare staff.

\section{Limitations}

This RCT was prematurely terminated. Numerous aspects of the ED environment were challenging to navigate during the pandemic, e.g. the uncertain situation, risk regarding virus transmission, and increased need for personal protective equipment. Reliance on face-to-face procedures, alongside reduced flow of patients presenting to the ED after a traumatic event, meant we were unable to continue this study.

The study described to participants during the informed consent process was significantly larger in scale than the final terminated trial reported here. The planned trial-with a much larger sample-would have afforded greater protection of participants' privacy, particularly given the unique ED site. Owing to such considerations, the current data has not been made publicly available. Overall, lessons learned include the need for post-trauma interventions that are suitable for participants under pandemic conditions [20] i.e. remote recruitment/delivery.

\section{Abbreviations}

CTR: Clinical Trials Registration; ED: Emergency Department; PTSD: Posttraumatic stress disorder; RA: Research Assistant; RCT: Randomised Controlled Trial.

\section{Acknowledgements}

We thank the management and staff in the ED and ED walk-in center for supporting recruitment and Julia Widoff, Caterina Vannucci and Felicia Sundström for support with data collection.

\section{Authors' contributions}

EAH conceived of the study including design with support from LS and MK; EAH and MK supervised and trained research assistants, and liaised with the hospital; LS, MK, BG and EAH, wrote the manuscript first draft; LS monitored electronic platform data, and created figure/table; KG provided nursing expertise during the study preparation and throughout data collection and write up; LI contributed to general intellectual work, study design and materials; MLM contributed to writing and conceptual interpretation; RST approved the 
statistical plan and provided power calculation. All authors read and approved the final manuscript.

\section{Funding}

Open access funding provided by Uppsala University. The project was mainly supported by the Swedish Research Council (2017-00957). EAH also receives funding from The Oak Foundation (OCAY-18-442). MK was supported by a FO Medical Psychology grant from Karolinska Institutet and Karolinska University Hospital. LS was supported by the Swiss National Science Foundation (P2BEP1_184378) and a Thunberg Fellowship by the Swedish Collegium for Advanced Study. KG was supported by a grant from FO Akut, Karolinska University Hospital. LI was supported by National Institute for Health Research (NIHR) Research Capability Funding.

\section{Availability of data and materials}

Data related to delivery of study procedures, recruitment, treatment adherence, outcome completion and attrition are reported within this article. Individual data for these 16 participants are not publicly available owing to considerations regarding the protection of participants' privacy. Study materials may be made available upon reasonable request with an appropriate materials transfer agreement (MTA) with Uppsala University.

\section{Declarations}

\section{Ethics approval and consent to participate}

Participants gave their written and informed consent to participate in the study. Ethical approval for this study was granted by Regional Research Ethics Committee, Stockholm, approval number 2019-05380.

\section{Consent for publications}

Not applicable.

\section{Competing interests}

EAH reports serving on the board of trustees of the charity MQ: Transforming Mental Health but receives no remuneration for this role. EAH receives royalties from books and occasional fees for workshops and invited addresses; receives occasional consultancy fees from the Swedish agency for health technology assessment and assessment of social services. LI is on the British Association for Behavioural and Cognitive Psychotherapies Scientific Committee and via this role is funded to attend the Annual Conference. The other authors have no conflicts of interests to declare.

\section{Author details}

'Division of Psychology, Department of Clinical Neuroscience, Karolinska Institutet, 17177 Stockholm, Sweden. ${ }^{2}$ Functional Area Medical Psychology, Karolinska University Hospital, Stockholm, Sweden. ${ }^{3}$ Department of Psychology, Uppsala University, Box 1225, 75142 Uppsala, Sweden. ${ }^{4}$ Swedish Collegium for Advanced Study, Uppsala, Sweden. ${ }^{5}$ Emergency and Reparative Medicine Theme, Karolinska University Hospital, Stockholm, Sweden. ${ }^{6}$ Department of Medicine Solna, Karolinska Institutet, Stockholm, Sweden. ${ }^{7}$ MRC/CSO Social and Public Health Sciences Unit \& Robertson Centre for Biostatistics, Institute of Health and Well Being, University of Glasgow, Glasgow, UK. ${ }^{8}$ Department of Psychiatry, University of Oxford, Oxford, UK. ${ }^{9}$ School of Psychology, The University of New South Wales, UNSW Sydney, Australia.

Received: 18 October 2020 Accepted: 15 April 2021

Published online: 10 May 2021

\section{References}

1. Kanstrup M, Singh L, Goransson KE, Widoff J, Taylor RS, Gamble B, et al. Reducing intrusive memories after trauma via a brief cognitive task intervention in the hospital emergency department: an exploratory pilot randomised controlled trial. Transl Psychiatry. 2021;11(1):30.
2. Kupfer DJ, Regier DA. Neuroscience, clinical evidence, and the future of psychiatric classification in DSM-5. Am J Psychiatry. 2011;168:172-4.

3. Iyadurai L, Visser RM, Lau-Zhu A, Porcheret K, Horsch A, Holmes EA, et al Intrusive memories of trauma: a target for research bridging cognitive science and its clinical application. Clin Psychol Rev. 2019;69:67-82.

4. American Psychiatric Association. Diagnostic and statistical manual of mental disorders: DSM-5. Fifth edition. ed: Arlington: American Psychiatric Publishing; 2013.

5. Singh L, Espinosa L, Ji JL, Moulds ML, Holmes EA. Developing thinking around mental health science: the example of intrusive, emotional mental imagery after psychological trauma. Cogn Neuropsychiatry. 2020;54:1-16.

6. Iyadurai L, Blackwell SB, Meiser-Stedman R, Watson PC, Bonsall MB, Geddes JR, et al. Preventing intrusive memories after trauma via a brief intervention involving Tetris computer game play in the emergency department: a proof-of-concept randomized controlled trial. Mol Psychiatry. 2018;23:674-82.

7. McNally RJ. Networks and nosology in posttraumatic stress disorder. JAMA Psychiat. 2017;74(2):124-5.

8. Visser RM, Lau-Zhu A, Henson RN, Holmes EA. Multiple memory systems, multiple time points: how science can inform treatment to control the expression of unwanted emotional memories. Philos Trans R Soc Lond B Biol Sci. 2018;373:1742

9. Monfils $\mathrm{MH}, \mathrm{Holmes}$ EA. Memory boundaries: opening a window inspired by reconsolidation to treat anxiety, trauma-related, and addiction disorders. Lancet Psychiatry. 2018;5(12):1032-42.

10. Sveriges Radio AB. Filosofiska Rummet: Vägen till en svensk filosofi: Sveriges Radio; 2018. Podcast: 20 May. https://sverigesradio.se/avsnitt/10735 96

11. Rothbaum BO, Kearns MC, Price M, Malcoun E, Davis M, Ressler KJ, et al. Early intervention may prevent the development of posttraumatic stress disorder: a randomized pilot civilian study with modified prolonged exposure. Biol Psychiatry. 2012;72(11):957-63.

12. Carlisle BG. Clinical trials stopped by Covid-19. 2020 https://covid.bgcar lisle.com/. https://covid19.bgcarlisle.com/

13. van Dorn A. COVID-19 and readjusting clinical trials. Lancet. 2020;396(10250):523-4.

14. Blendal R. Randomization: SmartTrial Help Center 2019 [12-10-2020]. https://help.smart-trial.co/display/HELPPlayground/Randomization.

15. Horsch A, Vial Y, Favrod C, Harari MM, Blackwell SE, Watson P, et al. Reducing intrusive traumatic memories after emergency caesarean section: A proof-of-principle randomized controlled study. Behav Res Ther. 2017;94:36-47

16. Kanstrup M, Rudman A, Göransson K, Andersson E, Olofsdotter Lauri K,

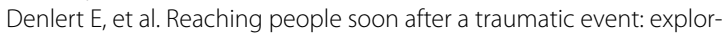
ing the feasibility of delivering a brief behavioural intervention in the emergency department to prevent intrusive memories of trauma. Under review.

17. Button KS, loannidis JPA, Mokrysz C, Nosek BA, Flint J, Robinson ESJ, et al. Power failure: why small sample size undermines the reliability of neuroscience. Nat Rev Neurosci. 2013;14(5):365-76.

18. Stein MB, Kerridge C, Dimsdale JE, Hoyt DB. Pharmacotherapy to prevent PTSD: results from a randomized controlled proof-of-concept trial in physically injured patients. J Trauma Stress. 2007;20:923-32.

19. National Institute for Health and Care Excellence. Posttraumatic Stress Disorder (NICE Guideline 116) 2018. https://www.nice.org.uk/guidance/ ng116.

20. Holmes EA, O'Connor RC, Perry VH, Tracey I, Wessely S, Arseneault L, et al. Multidisciplinary research priorities for the COVID-19 pandemic: a call for action for mental health science. Lancet Psychiatry. 2020;7(6):547-60.

\section{Publisher's Note}

Springer Nature remains neutral with regard to jurisdictional claims in published maps and institutional affiliations. 